\title{
Evaluating Diagnostic Efficacy of Cone Beam Computed Tomography Performed in Different Imaging Conditions Based on Field of View and Resolution in the Detection of Mandibular Condyle Erosions: An In Vitro Study
}

\author{
Sanaz Sharifi Shoshtari ${ }^{1}$, Mahshid Razavi ${ }^{1}$, Parisa Boostani Fard ${ }^{2,}{ }^{*}$, Ali Rohani ${ }^{2}$, Shadi Hamidiaval ${ }^{3}$ \\ and Naghme Niroomand ${ }^{4}$ \\ ${ }^{1}$ Department of Oral and Maxillofacial Radiology, School of Dentistry, Ahvaz Jundi Shapur University of Medical Sciences, Ahvaz, Iran \\ ${ }^{2}$ School of Dentistry, Ahvaz Jundi Shapur University of Medical Sciences, Ahvaz, Iran \\ ${ }^{3}$ Department of Oral and Maxillofacial Radiology, Lorestan University of Medical Sciences, Khoramabad, Iran \\ ${ }^{4}$ Department of Oral and Maxillofacial Radiology, Arak University of Medical Sciences, Arak, Iran \\ "Corresponding author: School of Dentistry, Ahvaz Jundi Shapur University of Medical Sciences, Ahvaz, Iran. Email: alirohani172@gmail.com
}

Received 2017 August 19; Revised 2019 May 02; Accepted 2019 May 07.

\begin{abstract}
Background: New cone beam computed tomography (CBCT) devices have the ability to take images with different fields of views (FOVs) and resolutions. The larger the FOV and the higher resolution of the image, the higher the patient's dose would be.

Objectives: This research aims to analyze the diagnostic accuracy of FOV's different dimensions and the various resolutions in the diagnosis of mandibular condyle erosions.

Materials and Methods: CBCT images using NewTom VGi (Verona, Italy) system in five different FOVs and resolutions (voxel size) were taken from eight human dried mandibles in which the erosion-like lesions were created on both condyles. Imaging was performed before and after the formation of erosion; afterwards, the images were evaluated by two maxillofacial radiologists to analyze the absence or presence of the lesions. Resultant data was evaluated by SPSS V. 22.0, McNemar and Kappa statistical methods.

Results: The highest sensitivity, specificity, positive predictive value (PPV), negative predictive value (NPV), and accuracy were attributed to $8 \times 8$ FOV and the lowest was attributed to $12 \times 8$ FOV. In analyzing different resolutions, the highest sensitivity, specificity, PPV, and NPV were attributed to high resolution ( 0.125 and $0.15 \mathrm{~mm}$ voxel size). There was no statistically significant difference between sensitivity, specificity, PPV, and NPV of different FOVs and different resolutions. Inter-observer and intra-observer cofficiency were at excellent range.

Conclusion: Considering there was no significant difference between the results, it is possible to use voxels in larger size to reduce patient's dose in order to detect mandibular condyle erosions. In addition, FOV with lower patient's dose is the method of choice.
\end{abstract}

Keywords: Mandibular Condyle Erosions, Cone Beam Computed Tomography (CBCT), Voxel Size, Field of View (FOV)

\section{Background}

Imaging is a significant diagnostic addition to the clinical evaluation of dental patients. With the increasing array of imaging modalities, dental radiology plays an innovative role in forming the diagnosis, treatment plan, and it has a prognostic value (1). Temporomandibular disorder (TMD) is the most common maxillofacial disorder which affects the natural operation of the joints. Between 28 to 82 percent of adults have the clinical signs (2). The first and the most common degenerative change of temporomandibular joint (TMJ) is erosion which shows the joint's instability.
In radiography, erosion is a local radiolucent area in the condyle accompanied with the density reduction of the bone's cortical level (3). Panogramic radiography, submentovortex, trans-cranial, trans-pharyngeal, lateral cephalometry, and also conventional tomography, computed tomography, and magnetic resonance imaging (MRI) are all the imaging methods that are being used for temporomandibular in-detail analysis. Newer techniques include cone beam computed tomography (CBCT), ultrasonography, and rapid prototype (RP) (4). However, interpretation of $2 \mathrm{D}$ images is hard because of the overlapping and superimposition of different structures (5). Recently, 
CBCT has been developed for maxillofacial regions and has been accepted and prevalent in dentistry profession and it provides reconstructed images with high diagnostic quality using the lower absorbed dose and the shorter scan time compared to CT examinations (6). During the CBCT scan, the radiated ray is affected by exposure parameters including voxel size, field of view (FOV) dimensions, rotation degree, tube's current and voltage (7).

FOV is one of the parameters (8). Using a smaller FOV causes the increase of image resolution by the decrease of fading and scattering radiation (9). Also, it is important to mention that these new devices have the capability of imaging with different voxel sizes (different resolutions); however, the higher the resolution, the higher dose would be applied to the patient (10).

\section{Objectives}

Only a handful of studies have been performed on FOV's different dimensions $(11,12)$. As there exists different FOVs and imaging capabilities with different resolutions in the common CBCT devices, the present research aims to analyze the effect of FOV's different dimensions and also the device's different resolutions with the same FOVs in the evaluation of one of the most common temporomandibular disorders.

\section{Materials and Methods}

In this experimental (in vitro) study, similar to a study conducted by Librizzi et al. (13), in the first step, eight samples of human dry mandible were collected and sterilized. They tried to collect samples that did not have any erosion or broken parts in condyle; however, in the cases where some erosion already existed on samples, in order to reach an exact result and prevent the errors while observing the provided images, the conditions of condyles were recorded and they were reported to the observers. Considering the difficulty of collecting samples, based on the statistics consultant's idea, it was decided that the imaging of each mandible should be done in two conditions: the first condition entailed the normal and the intact condyles and the second condition consisted of images after erosion simulation on the condyle. In the first step, the imaging process of all of the normal samples (without erosion) was started through CBCT device. Then, during the imaging process through CBCT (NewTom, VGi, Italy), the mandible was put in an optimal position using adhesive tape and was fixed after setting the light lines. The device we used in our study was compatible with selecting denture scan mode, instead of using a patient scan mode for the reconstruction of soft tissue. The samples were scanned with different FOVs and resolutions in CBCT. The FOVs and resolution were predefined in the CBCT device, and they were as follows:

1. $6 \times 6$ FOV and high resolution (voxel size 0.150 )

$2.8 \times 8$ FOV and high resolution (voxel size 0.125 )

3. $8 \times 12$ FOV and high resolution (voxel size 150)

4. $8 \times 8$ FOV and regular resolution (voxel size 0.25 )

5. $8 \times 12$ FOV and regular resolution (voxel size 0.3 )

The device's exposure condition was 110 KVP and 27.07 MAS. CBCT provided images that were evaluated on a computer. After making sure about the imaging result, each image, which was similar to the related sample's code, was coded. Next, the reconstructed images were provided from the imaged samples using NNT Software (thickness $=0.5$ $\mathrm{mm}$, step =1 mm); finally, it was stored on a DVD. In order to simulate erosion on condyles, holes were made on the anterior pole of the condyles, using a high speed, round bur hand piece. Erosion size was equal to round bur's diameter $(0.1 \mathrm{~mm})$ and its depth was equal to half of the round bur's diameter $(0.5 \mathrm{~mm})$. In the next step, all of the readymade samples were put in the process of CBCT imaging (with the same above-mentioned condition) and the constructed images were stored on a DVD. Therefore, all of the 16 condyles (with erosion and also without erosion), were exposed five times, and finally 160 imaging volumes were prepared and we made multiplanar images from those volumes (Figures 1-3).

The coding process of the images provided from eight normal samples was different from the coding process of the other eight samples in which the erosion was simulated in the condyle. In the last stage, the provided images were given to two observers. Our observers were maxillofacial radiologists who had at least 5 years of experience in evaluation of CBCT images. They observed each image separately. They were not informed of the presence or absence of the condyle erosions, or the number of the samples with and without condyle erosion; also the sequence of images that were given to the observers was random and in a way that each observer was evaluating the images separately in a dim room at a specific time of the day (equal condition for the light) using a Sony 14-inch LED flat-screen monitor with $800 \times 1280$ resolution.

Observers were allowed to manipulate images in terms of contrast, brightness, and magnification. Also, they were allowed to evaluate images in all multiplanar views. They were asked to mark the presence or absence of erosion in condyle as 0 (absence) and 1 (presence). Each score given by the observers was recorded as a sample unit. Then, after a month, the observers had to observe and record them again. Finally, all of the results were given for statistical as- 


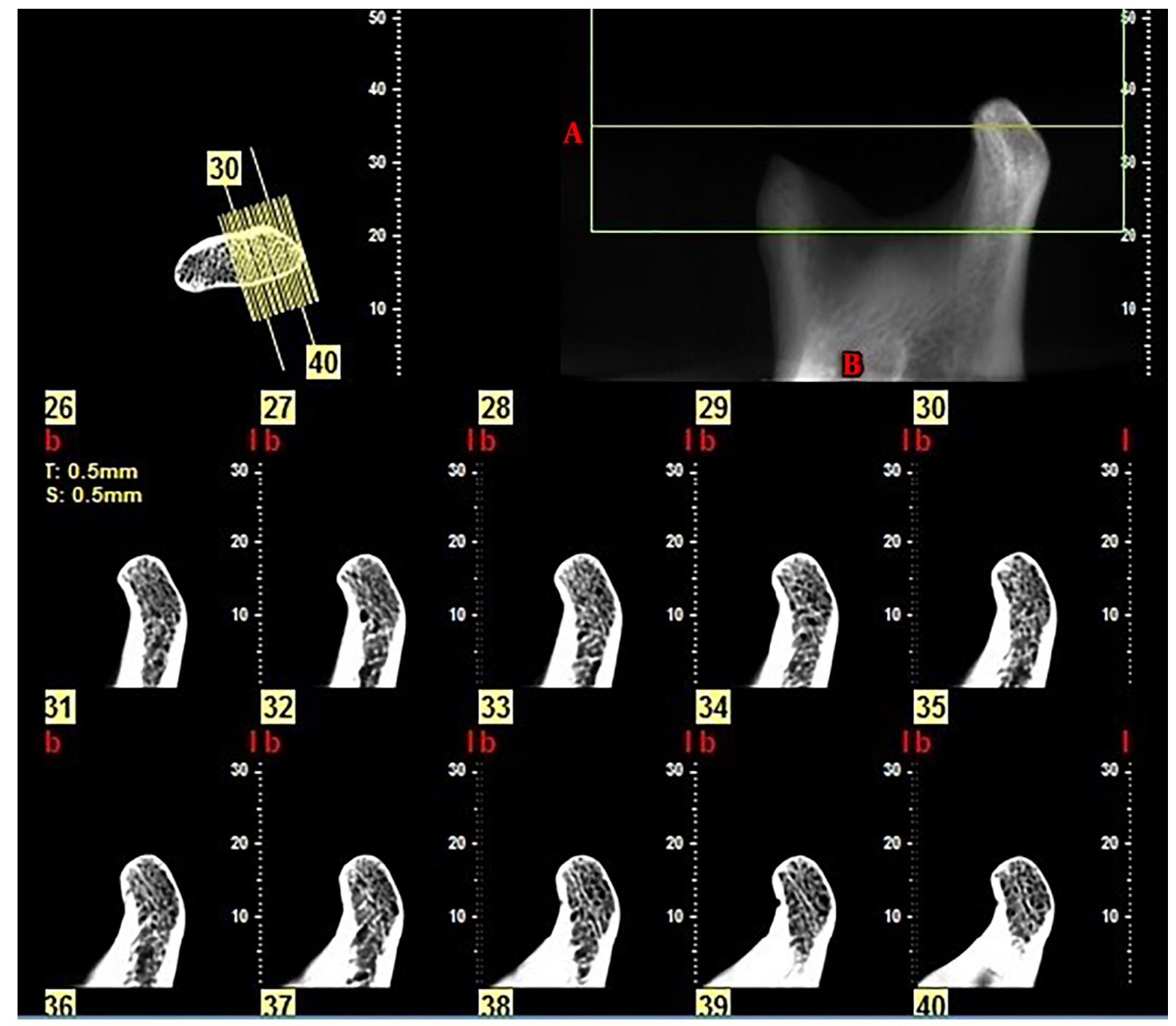

Figure 1. Cone beam computed tomography of dry mandibular condyle (mediolateral view)

sessment which included SPSS V. 22.0 (IBM Corp. Released 2013. IBM SPSS Statistics for Windows, Version 22.0. Armonk, NY: IBM Corp), McNemar and Kappa.

\section{Results}

\subsection{Analyzing FOVs and Different Resolutions}

Five modes of $6 \times 6$ FOV and high resolution, $8 \times 8$ FOV and high resolution, $8 \times 12$ FOV and high resolution, $8 \times$ 8 FOV and regular resolution, and $8 \times 12$ FOV and regular resolution were analyzed and compared (Tables 1 - 3). Furthermore, in order to analyze and compare the different resolutions, the following modes were analyzed and compared with each other: $8 \times 8$ FOV and regular resolution (voxel size 0.25 ) with $8 \times 8$ FOV and high resolution (voxel size 0.125 ), $12 \times 8$ FOV and regular resolution (voxel size 0.3 ) with $12 \times 8$ FOV and high resolution (voxel size 0.150 ). In general, the ability to detect mandibular condyle erosions in high resolution modes and regular resolution modes were compared with each other.

Results of FOV's sensitivity, specificity, positive predictive value (PPV), and negative predictive value (NPV) for all of the observers were reported. FOV and different resolution's receiver operating characteristics (ROC) curve is illustrated in Figures 4 and 5. The area under the ROC curve is shown in Table 2.

1. In the analysis of different FOVs, the highest sensitivity, specificity, PPV, and NPV was attributed to $8 \times 8$ FOV and 

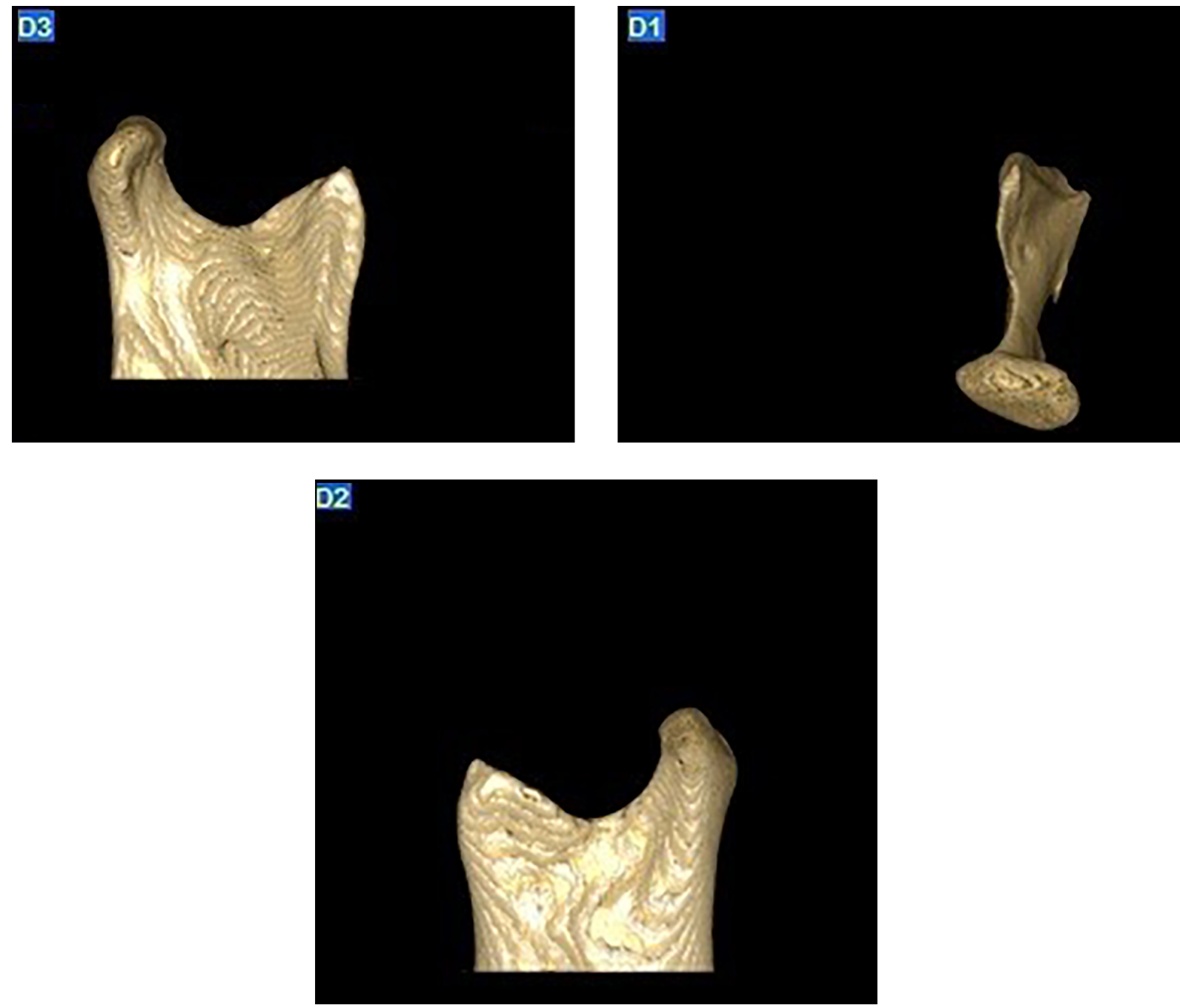

Figure 2. Cone beam computed tomography of dry mandibular condyle (3D reconstruction view)

the least amount was attributed to $12 \times 8$ FOV. Moreover, in the analysis of different resolutions, the highest sensitivity, specificity, PPV, and NPV was attributed to both $8 \times 8$ FOV and high resolution and $12 \times 8$ FOV and high resolution and between these two high resolution modes, sensitivity, specificity, PPV, and NPV of $8 \times 8$ FOV were the highest.

2. By taking ROC curve into consideration, the largest area which is under the curve (the highest accuracy) was related to $8 \times 8$ FOV. In addition, in the analysis of resolution, the highest accuracy was related to the higher resolutions and between these two high resolution modes, $8 \times 8$ FOV had the higher accuracy. However, there was no statistically significant difference between sensitivity, specificity, PPV, and NPV between different FOVs and different resolutions.
3. Inter-observer and intra-observer cofficiency were at excellent range.

\section{Discussion}

The objective of the present research was to evaluate different aspects and dimensions of FOV and different resolutions of CBCT for the diagnosis of mandibular condyle erosions. The aim was to find a solution for decreasing patient's absorbed dose together with preserving the image quality and diagnostic value.

One of the goals of this research was to analyze whether the increase of voxel size causes trouble in the diagnosis of mandibular condyle erosions. If no, some measurements should be done to detect mandibular condyle 


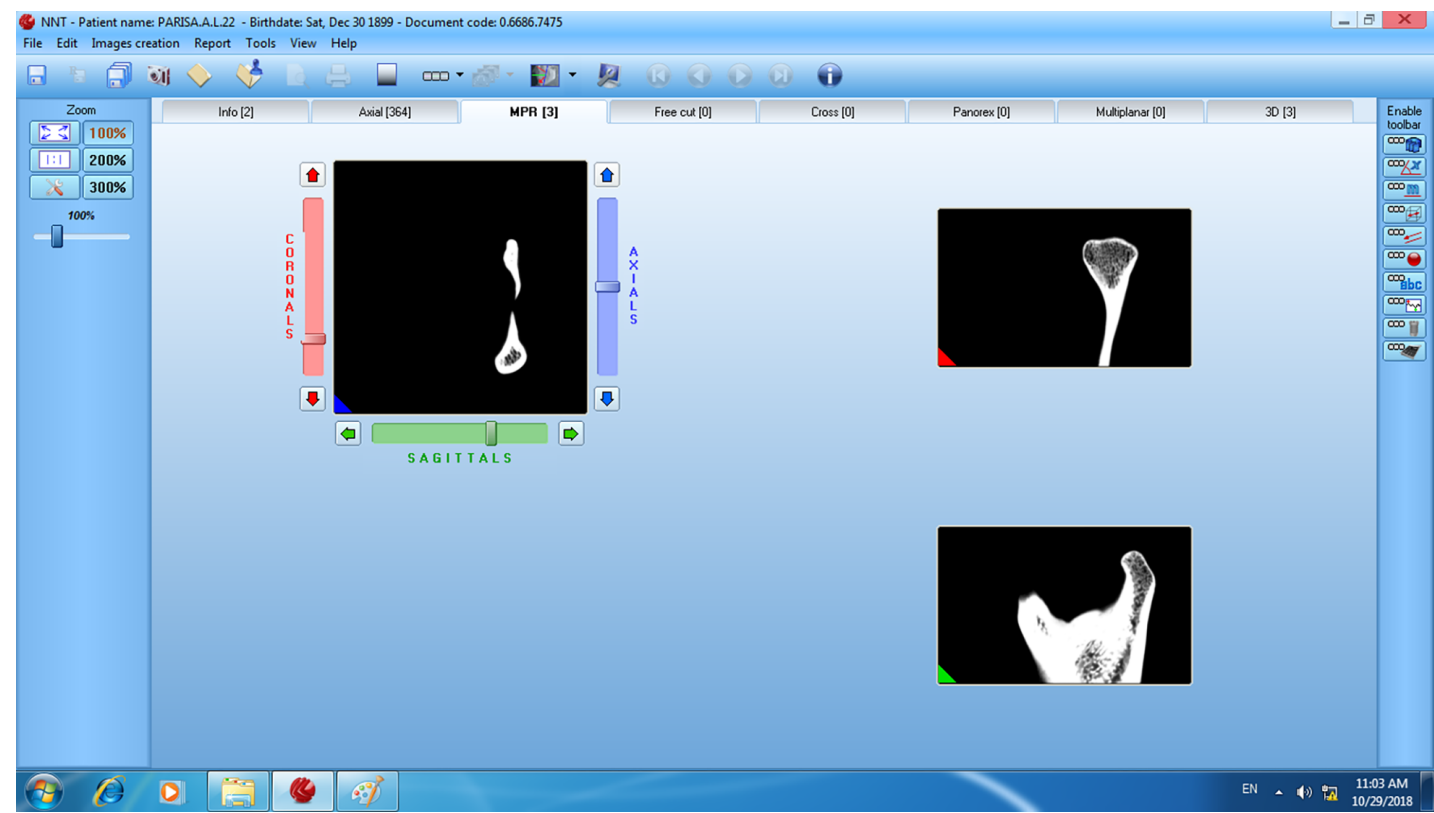

Figure 3. Cone beam computed tomography of dry mandibular condyle (mediolateral view)

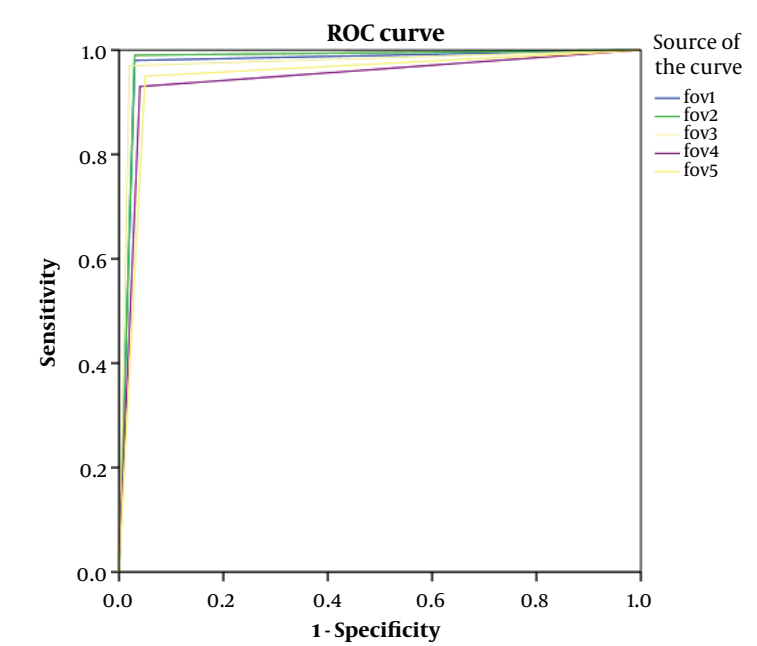

Figure 4. ROC curves of different conditions of cone beam computed tomography (in terms of FOV and resolution) in detection of mandibular condyle erosion

erosions by increasing voxel size, and consequently decreasing the received dose by the patient in CBCT examinations.

Based on this study, there was no significant difference between high resolution modes (voxel size 0.125 and 0.15 ) and the methods using regular resolution (voxel size 0.25 and 0.3). However, the best diagnostic power was related

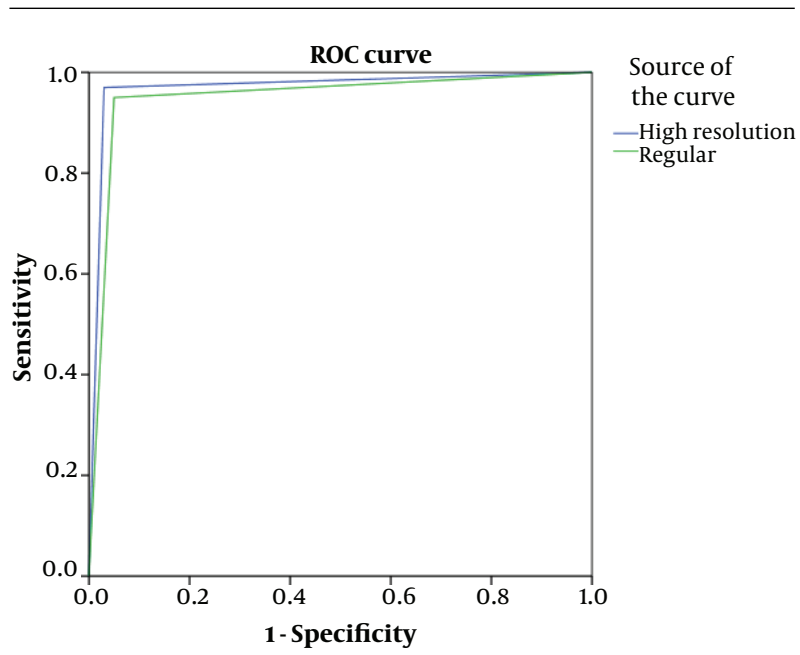

Figure 5. ROC curves of different resolutions of cone beam computed tomography (high versus regular) in detection of mandiular condyle erosion

to FOV $8 \times 8$. Most likely, this is due to voxel size, which among the five different modes has the minimum voxel size. Yet, since there is no statistically significant difference, it is possible to use regular resolutions instead of higher resolutions, which accordingly decreases the patient's absorbed dose and at the same time has an optimum diagnostic value. 


\begin{tabular}{|c|c|c|c|c|}
\hline & Sensitivity (\%) & Specificity (\%) & PPV (\%) & NPV (\%) \\
\hline $6 \times 6$ FOV and high resolution & 98 & 97 & 97.02 & 97.97 \\
\hline $8 \times 8$ FOV and high resolution & 99 & 99 & 99 & 99 \\
\hline $8 \times 12$ FOV and high resolution & 97 & 96.7 & 97 & 97.02 \\
\hline $8 \times 8$ FOV and regular resolution & 93 & 96 & 98.87 & 93.2 \\
\hline $8 \times 12$ FOV and regular resolution & 95 & 95 & 95 & 95 \\
\hline High resolution & 98 & 98 & 98 & 98 \\
\hline Regular resolution & 94 & 95.5 & 95.43 & 94.08 \\
\hline
\end{tabular}

Abbreviations: FOV, field of view; NPV, negative predictive value; PPV, positive predictive value.

${ }^{\mathrm{a}}$ Values are expressed as percentage.

\begin{tabular}{lccccc}
\hline Table 2. Area Under the ROC Curve of Cone Beam Computed Tomography in Detection of Mandibular Condyle Erosion in Different FOVs and Resolutions \\
\hline $\begin{array}{l}\mathbf{6} \times \mathbf{6} \text { FOV and } \\
\text { high resolution }\end{array}$ & $\begin{array}{c}\mathbf{8} \times \mathbf{8} \text { FOV and } \\
\text { high resolution }\end{array}$ & $\begin{array}{c}\mathbf{8} \times \mathbf{1 2} \text { FOV and } \\
\text { high resolution }\end{array}$ & $\begin{array}{c}\mathbf{8} \times \mathbf{8} \text { FOV and } \\
\text { regular } \\
\text { resolution }\end{array}$ & $\begin{array}{c}\mathbf{8} \times \mathbf{1 2} \text { FOV and } \\
\text { regular } \\
\text { resolution }\end{array}$ & $\begin{array}{c}\text { High } \\
\text { resolution }\end{array}$ \\
\hline $\begin{array}{l}\text { The area under } \\
\text { the ROC curve }\end{array}$ & 0.975 & 0.980 & 0.975 & 0.945 & 0.945 \\
resolution
\end{tabular}

Abbreviations: FOV, field of view ; ROC, receiver operating characteristic.

Table 3. Comparison of Different FOVs and Resolutions of Cone Beam Computed Tomography in the Detection of Mandibular Condyle Erosions

\begin{tabular}{|c|c|}
\hline FOVs and resolutions & Pvalue \\
\hline Group 1 versus group 2 & 1 \\
\hline Group 1 versus group 3 & 0.727 \\
\hline Group 1 versus group 4 & 0.454 \\
\hline Group 1 versus group 5 & 1 \\
\hline Group 2 versus group 3 & 0.453 \\
\hline Group 2 versus group 4 & 0.302 \\
\hline Group 2 versus group 5 & 0.791 \\
\hline Group 3 versus group 4 & 0.804 \\
\hline Group 3 versus group 5 & 1 \\
\hline Group 4 versus group 5 & 0.375 \\
\hline High resolution versus regular resolution & 0.577 \\
\hline
\end{tabular}

Abbreviations: FOV, field of view; 1, $6 \times 6$ FOV and high resolution; $2,8 \times 8$ FOV and high resolution; $3,8 \times 12$ FOV and high resolution; $4,8 \times 8$ FOV and regular resolution; 5, $8 \times 12$ FOV and regular resolution.

Since the sensitivity difference of all modes was not statistically significant, it could be concluded that CBCT with any FOV and resolution is a suitable technique to diagnose mandibular condyle erosions. It is possible to consider CBCT as a suitable technique for the diagnosis of mandibular condyle erosions, even in cases in which the resolution is lower (larger voxel sizes); the results, nonetheless, could be trusted.

When the specificity is in lower level, because the false positive is higher, there is the probability of a wrong diagnosis. Due to low specificity, the normal mandibular condyle is reported to have erosion and the therapeutic process would be distorted. Considering the findings which show the specificity differences in high resolution modes (voxel size 0.125 and 0.15) and the methods with regular resolutions (voxel size 0.25 and 0.3) and also different FOVs is not significant, even in the cases that the resolution is lower (larger voxel sizes), it is possible to ensure that the condyle is normal and does not have any problem.

In the present study, the high resolution modes $(8 \times 8$ FOV, voxel size $0.125 \mathrm{~mm}$ and $12 \times 8 \mathrm{FOV}$, voxel size 0.150 ) were related to the condition with the highest sensitivity, specificity, PPV, and NPV. Between these two high resolution modes, $8 \times 8$ FOV had the highest sensitivity, specificity, PPV, and NPV; however, it was not significant.

No other similar study in this field exists. Here some researches have been reviewed about the analysis of FOV and different resolutions in dentomaxillofacial problems.

Amintavakoli et al. have conducted a research entitled "The effect of CBCT voxel size on the diagnosis of vertical and horizontal root fractures: an in-vitro study". Results have shown that CBCT accuracy in voxel size under $300 \mathrm{mi}-$ crometer was better. The highest sensitivity, PPV, and accuracy was in 0.1 voxel size and the highest specificity and NPV was in $0.76 \mathrm{~mm}$ voxel size; however, it was not significant (12). The fact that voxel size does not have any effect on the detection of root fracture and the difference between voxel sizes were not significant, was in line with the result of the present research. 
Moreover, Ozer have analyzed the effect of voxel size $(0.4,0.3,0.2$, and 0.125$)$ on the diagnosis of vertical fractures of the root. Based on the results of their study, both 0.2 and 0.125 voxel sizes had more sensitivity and specificity although it was not significant (14). Considering the fact that the smallest voxel size was selected, the results are in line with the result of the present research. Although they were analyzing teeth problems (as opposed to our study which is about erosion in the condyle), different resolutions did not have any effect on the diagnosis of the problem.

In a study conducted by Liedke et al., $0.2,0.3$, and 0.4 voxel sizes were used for the root's external resorption analysis. Results indicate that there was no statistically significant difference between the voxel sizes. Nevertheless, according to their study, the best voxel size was 0.3 (15). Similar to our study, they concluded that there is no significant difference between different voxel sizes, but their size of choice was not the smallest one. Regarding the use of larger voxel size by Liekde (compared to the use of small voxel size in our study and Amintavakoli and Ozer), it seems the results they reached in their studies were due to the absence of small voxel size. Furthermore, their studies explored dental problems which is in contrast with our study evaluating the bony erosion.

Nikneshan et al. conducted a research entitled "Effect of voxel size on diagnosis of external root resorption defects using cone beam computed tomography". Artificial defects were prepared in the buccal and lingual surfaces of the roots and CBCT scans were obtained with different voxel sizes. Their results showed similar diagnostic efficacies of all voxel sizes. Accordingly, they suggest that larger voxel size could be used with adequate efficacy for diagnosis of root resorption with minimal patient's dose and the shortest scanning time (16). The result of their study is in line with the current study in which we suggest using larger voxel size to reduce patient's dose.

The present study has analyzed the effect of FOV's size on the diagnosis of mandibular condyle erosions. Among $6 \times 6$ FOV (voxel size 0.150), $8 \times 8$ FOV (voxel size 0.125), 8 $\times 12$ FOV (voxel size 0.150 ) with high resolution, the highest amount of sensitivity, specificity, PPV, NPV and accuracy was attributed to $8 \times 8$ FOV with high resolution. However, the difference between these FOVs was not significant. Because the settings of the device, $8 \times 8$ FOV with high resolution has the lowest voxel size which can have a great effect on the diagnosis of final image details.

Costa et al. conducted a research entitled "the application of large-volume cone-beam computed tomography in diagnosis and localization of horizontal root fracture in the presence and absence of intracanal metallic post" in Brazil. Findings have shown high accuracy in the group without metallic post which indicates a considerable statistical difference in the group which had metallic post. It was reported that CBCT with small FOV has high accuracy (73\% - 88\%) in the diagnosis of horizontal fractures of the root in cases that did not have metallic posts (17). The mentioned factor was in line with the result of the present research. However, no teeth problem was analyzed in this study and it is different from the present study in that it analyzed bone disorders. However, the result confirms the use of small FOV(i.e. it is not necessary to use the large FOV for the diagnosis of teeth problem) which is in line with the results of the present study.

Eskandarlou et al. conducted a research entitled "comparison between cone beam computed tomography and multislice computed tomography in diagnostic accuracy of maxillofacial fractures in dried human skull". In this research, they used a saw with $20 \mathrm{~mm}$ scalpel to create a small fracture in human dry skull. Results have shown that sensitivity, specificity, PPV, NPV, and CBCT diagnosis accuracy of $6 \times 6$ FOV was more than that of $15 \times 15$ FOV; however this difference was not significant (11). Although this study is about a different problem, it has a similar outcome with the present research. FOV's smaller size results in a more exact record of teeth or maxillofacial problem but there is no statistically significant difference.

It is obvious that almost all of the studies above could not find a significant difference between FOV and resolution. However, it is necessary to have more research in this field. It seems that it is not necessary to impose extra dose to the patient. Although CBCT with small voxel size and a high spatial resolution is suitable for diagnostic evaluations (including details like mandibular condyle erosions), since smaller voxel sizes result in higher patient dose and considering the fact that there is not a significant difference between the results of different voxel sizes and different FOVs, it is possible to detect mandibular condyle erosions with methods that decrease the patient's absorbed dose.

Our limitation in this study was finding a human cadaver. We suggest to other authors to evaluate this condition in vivo. Also, assessment of other FOVs is suggested for similar researches. Evaluation of other TMJ diseases is also recommended.

\section{Footnotes}

Authors' Contributions: Concept, design, manuscript editing and review: Sanaz Sharifishooshtari; literature search, statistical analysis: Mahshid Razavi and Ali Rohani; manuscript preparation: Parisa Boostanifard; experimental study: Parisa Boostanifard, Shadi Hamidiaval, and Naghme Niroomand 
Conflict of Interests: None declared.

Ethical Approval: IR.AJUMS.REC.1394.689.

Financial Disclosure: There is no financial disclosure.

Funding/Support: There is no funding/support.

\section{References}

1. Ghali SR, Katti G, Shahbaz S, Katti C. Cone beam computed tomography: A boon for maxillofacial imaging. J Indian Acad Oral Med Radiol. 2017;29(1):30. doi: 10.4103/jiaomr.JIAOMR_89_16.

2. Petrikowski C. Diagnostic imaging of the temporomandibular joint In: White SC, Pharoah MJ, editors. Oral radiology principle and interpretation. 6th ed. St. Louis, Missouri, USA: Mosby; 2009. p. 473-505.

3. Zamani Naser A, Shirani AM, Hekmatian E, Valiani A, Ardestani P, Vali A. Comparison of accuracy of uncorrected and corrected sagittal tomography in detection of mandibular condyle erosions: An exvivo study. Dent Res J (Isfahan). 2010;7(2):76-81. [PubMed: 22013461]. [PubMed Central: PMC3177372].

4. de Senna BR, dos Santos Silva VK, Franca JP, Marques LS, Pereira LJ. Imaging diagnosis of the temporomandibular joint: Imaging Diagnosis of the Temporomandibular Joint: Critical review of indications and new perspectives. Oral Radiol. 2009;25(2):86-98. doi: 10.1007/s11282-009-0025-x.

5. Saati S, Kaveh F, Yarmohammadi S. Comparison of cone beam computed tomography and multi slice computed tomography image quality of human dried mandible using 10 anatomical landmarks. J Clin Diagn Res. 2017;11(2):ZC13-6. doi: 10.7860/JCDR/2017/20637.9253. [PubMed: 28384972]. [PubMed Central: PMC5376866].

6. Hilgers ML, Scarfe WC, Scheetz JP, Farman AG. Accuracy of linear temporomandibular joint measurements with cone beam computed tomography and digital cephalometric radiography. Am J Orthod Dentofacial Orthop. 2005;128(6):803-11. doi: 10.1016/j.ajodo.2005.08.034. [PubMed: 16360924].

7. Loubele M, Bogaerts R, Van Dijck E, Pauwels R, Vanheusden S, Suetens $\mathrm{P}$, et al. Comparison between effective radiation dose of CBCT and MSCT scanners for dentomaxillofacial applications. Eur J Radiol. 2009;71(3):461-8. doi: 10.1016/j.ejrad.2008.06.002. [PubMed: 18639404].
8. White SC, Pharoah MJ. Oral radiology principle and interpretation. 3. 6th ed. Philadelphia: Elsevier; 2014. p. 149-567.

9. Daly MJ, Siewerdsen JH, Moseley DJ, Jaffray DA, Irish JC. Intraoperative cone-beam CT for guidance of head and neck surgery: Assessment of dose and image quality using a C-arm prototype. Med Phys. 2006;33(10):3767-80. doi:10.1118/1.2349687. [PubMed: 17089842].

10. Bushong SC. Radiologic science for technologists. 2. 9th ed. St. Louise: Mosby; 2013. 453 p.

11. Eskandarlou A, Poorolajal J, Talaeipour AR, Talebi S, Talaeipour M. Comparison between cone beam computed tomography and multislice computed tomography in diagnostic accuracy of maxillofacial fractures in dried human skull: An in vitro study. Dent Traumatol. 2014;30(2):162-8. doi: 10.1111/edt.12058. [PubMed: 23826823].

12. Amintavakoli N. The effect of cone beam CT voxel size on the identification of vertical and horizontal root fractures: An in-vitro study [dissertation]. Toronto: University of Toronto; 2013.

13. Librizzi ZT, Tadinada AS, Valiyaparambil JV, Lurie AG, Mallya SM. Cone-beam computed tomography to detect erosions of the temporomandibular joint: Effect of field of view and voxel size on diagnostic efficacy and effective dose. Am J Orthod Dentofacial Orthop. 2011;140(1):e25-30. doi: 10.1016/j.ajodo.2011.03.012. [PubMed: 21724068].

14. Ozer SY. Detection of vertical root fractures by using cone beam computed tomography with variable voxel sizes in an in vitro model. J Endod. 2011;37(1):75-9. doi: 10.1016/j.joen.2010.04.021. [PubMed: 21146082].

15. Liedke GS, da Silveira HE, da Silveira HL, Dutra V, de Figueiredo JA Influence of voxel size in the diagnostic ability of cone beam tomography to evaluate simulated external root resorption. J Endod. 2009;35(2):233-5. doi: 10.1016/j.joen.2008.11.005. [PubMed: 19166780].

16. Nikneshan S, Valizadeh S, Javanmard A, Alibakhshi L. Effect of voxel size on detection of external root resorption defects using cone beam computed tomography. Iran J Radiol. 2016;13(3). e34985. doi: 10.5812/iranjradiol.34985. [PubMed: 27853499]. [PubMed Central: PMC5107261].

17. Costa FF, Gaia BF, Umetsubo OS, Pinheiro LR, Tortamano IP, Cavalcant MG. Use of large-volume cone-beam computed tomography in identification and localization of horizontal root fracture in the presence and absence of intracanal metallic post.JEndod.2012;38(6):856-9. doi: 10.1016/j.joen.2012.03.011. [PubMed: 22595127]. 